Relations industrielles

Industrial Relations

\title{
Le service social dans l'entreprise, par Madeleine Bernard, Éditions du Centurion, Socioguides, Paris, 1966, 204 pages.
}

\section{André Petit}

Volume 22, numéro 4, 1967

URI : https://id.erudit.org/iderudit/027846ar

DOI : https://doi.org/10.7202/027846ar

Aller au sommaire du numéro

Éditeur(s)

Département des relations industrielles de l'Université Laval

ISSN

0034-379X (imprimé)

1703-8138 (numérique)

Découvrir la revue

Citer ce compte rendu

Petit, A. (1967). Compte rendu de [Le service social dans l'entreprise, par

Madeleine Bernard, Éditions du Centurion, Socioguides, Paris, 1966, 204 pages.]

Relations industrielles / Industrial Relations, 22(4), 583-584.

https://doi.org/10.7202/027846ar

Tous droits réservés @ C Département des relations industrielles de l'Université Laval, 1967
Ce document est protégé par la loi sur le droit d'auteur. L'utilisation des services d'Érudit (y compris la reproduction) est assujettie à sa politique d'utilisation que vous pouvez consulter en ligne.

https://apropos.erudit.org/fr/usagers/politique-dutilisation/ 
trielles dans différentes régions comparables au Chili.

Le haut niveau acodémique de cette publication ne fait aucun doute. L'auteur est un professeur de I'Université Cornell où il donne, depuis 1963, un cours sur \& Les Relations Industrielles en Amérique Latine $\gg$. Depuis 1958, il eut très souvent l'occasion de visiter le Chili et d'y travailler, soit comme invité, soit comme membre d'un projet conjoint d'études entre I'Université du Chili (Santiago) et I'Université Cornell. Le volume $a$, de plus, été soumis aux commentaires de nombreux intellectuels, pour la plupart spécialistes du système de relations industrielles de l'Amérique Latine, et a été corrigé collectivement

L'effort de compréhension des Etats-Unis envers l'Amérique Latine est généralement considéré comme insuffisant. Cette publication fait vraisemblablement exception. Elle reflète la qualité et mérite certainement d'être lue.

André PETIT

La conduite des réunions, la formation permanente en sciences humaines sous la direction de Roger Mucchielli, Librairies techniques / Entreprise Moderne d'Edition, Paris, avril 1967, 130 pages.

Cet ouvrage est divisé en deux parties: l'une, intitulée a connaisance du problème $*$, est une partie d'information théorique et de connaissances intellectuelles indispensables sur le thème proposé. L'autre, intitulée " exercices pratiques $»$, est une partie de formation personnelle concrète, présentée de manière progressive.

A la fin de la première partie se trouve un lexique des mots techniques utilisés. A la fin de la seconde, se trouvent des types de programmes pour sessions de perfectionnement de groupe.

La première partie est de beaucoup la plus intéressante et captivante. Le titre des cinq exposés parlent d'eux-mêmes: "Les problèmes psychologiques posés par la conduite des réunions et par la participation , «Parler à un public " Animer un grand groupe », "Le brainstorming », "La conduite des réunions de petit groupe . Chacun de ces exposés suggère des méthodes pour contourner les difficultés inhérentes à ces sujets et en arriver ainsi dे une plus grande efficacité

C'est au centre du livre que le lecteur intéressé par une formation personnelle, trou- vera l'ordonnancement rationnel de cette formation, mettant en oeuvre, parallèlement, les travaux pratiques et l'information. II y verra l'organisation pédagogique de son auto-formation.

Toutefois, cet ouvroge est surtout destiné à tous ceux qui ont ou qui auront o animer une réunion.

\section{Pierre DIONNE}

Le service social dans l'entreprise, par Madeleine Bernard, Editions du Centurion, Socioguides, Paris, 1966, 204 pages.

L'évolution moderne tend à donner, à l'intérieur des entreprises, une place de plus en plus large aux disciplines humaines. Les cadres de l'entreprise, pour faire foce aux situations nouvelles, présentent des qualifications académiques de plus en plus diversifiées. Des gens de profession qu'on considérait autrefois comme exclus de l'entreprise, y trouvent de plus en plus leur place. II semble que ce soit le cas, du moins en France, pour les gens du Service Sociol.

L'auteur, qui a eu elle-même une expérience dans l'entreprise veut aider à définir et à faire connaître cette dimension nouvelle de la profession. Elle nous renseigne donc d'abord sur les différentes responsabilités que peuvent assumer, à l'intérieur de l'entreprise, les gens de sa profession, tout en insistant sur le caractère essentiel d'évolution et de transformation de cette tâche dans un univers technique et social en voie de profond changement. Une vue lucide de la société, telle qu'elle existe, l'amène d̀ traiter des conditions d'insertion de I'assistante sociale dans l'entreprise.

Le service social, qu'on avait tendance à considérer autrefois comme une * vocation * et qu'il faut de plus en plus considérer comme un métier ou une profession est en droit, au même titre que les autres professions de s'interroger sur so propre évolution. Cette discipline est encore jeune, puisqu'il semble que ce travail soit le premier à tenter de faire l'analyse d'une tâche spécialisée de service social. Cette nouvelle dimension professionnelle, Mlle Bernard en donne les conditions d'existence et de développement. Elle termine d'ailleurs par une extrapolation sur le service social d'entreprise dans le monde.

$\mathrm{Ce}$ volume présente donc un intérêt évident pour les gens de service social. II présente aussi de l'intérêt pour n'importe quel 
outre professionnel, (qui o le devoir de se tenir ou courant) et surtout pour ceux qui ò l'intérieur de l'entreprise, pourront entrer en contact ou collaborer avec ces nouveaux spécialistes.

\section{André PETIT}

* Le prix du progrès », Chronique sociale de France, Cahier \# 2, mai 1967, 136 pages

* Il semble parfois que nos sociétés occidentales ne sachent plus elles-mêmes ò quoi elles destinent leurs richesses ou leur puissance. Quel est le sens de l'histoire que nous sommes en train de vivre? De quel prix payons-nous le Progrès dans notre civilisation? Comment orienter le développement économique à l'épanouissement de I'homme et non d̀ son asservissement? Une revue comme la Chronique Sociale ne peut esquiver de telles questions. Ce numéro leur est consacré dans le but de mettre à la portée de plus de gens possibles les contributions les plus importantes et les plus originales d'auteurs français et étrangers à la recherche sur le sens de notre histoire.

Bernard Cazes rappelle dans un premier article les principaux problèmes que pose la répartition des gains de la croissance économique dans nos sociétés.

Le professeur Weisskoff met ensuite en lumière le coût psychologique de la civilisation industrielle, l'insécurité intérieure si coractéristique de l'homme moderne doté par ailleurs de si nombreux moyens pour garantir so sécurité externe.

Le professeur Wilkinson, philosophe et physicien, après avoir analysé à travers I'histoire la lutte de l'homme pour survivre dे une matérialisation croissante, rappelle pourquoi dans un monde en explosion brutale et dans une société de plus en plus quantitative il importe plus que jamais de sauver les valeurs humaines.

Le docteur Dubos, spécialiste de la microbiologie et de la pathologie expérimentale, présente ses réflexions sur l'influence de la science sur la culture moderne et les possibilités offertes ò la science de détruire ou de transformer tous les aspects de la vie humaine

Enfin, Bertram Gross plaide pour que nous acceptions de retenir comme indice du Progrès non seulement les données quantitotives de l'économie mais oussi des critères qualitatifs tels que le progrès des arts, de la culture et de la participation démocro- tique de choque citoyen à lo vie de leur pays.

Il revient à Joseph Folliet de rappeler lo nécessité d'une sagesse, même ou surtout, dans le monde des ordinateurs.

* C'est de cette sagesse en effet que dépendro notre copacité d'introduire dans lo société quantitative des valeurs telles que la justice et de programmer dans des ordinateurs de telles valeurs de façon à ce qu'elles ne puissent aisément en être délogées. Car de la permanence de ces valeurs dans nos sociétés industrielles dépend en définitive le Progrès de notre civilisation. »

\section{Pierre DIONNE}

\section{Determination of the Appropriate Bargain- ing Unit, by Labour Relations Boards in Canada, par Edward E. Herman Canada Deportment of Labour, Econornics and Research Branch, November 1966, 227 po- ges.}

La détermination de l'unité de négociation est la responsabilité des différentes commissions des relations de travail. Au Conada, 11 de ces dites commissions existent -- 10 provinciales et 1 fédérale. L'auteur tente, dans le présent ouvrage, de faire une étude comparative détaillée des pratiques de ces commissions, ce qui n'avait pas encore été réalisé

Le but de cette étude est d'examiner les différentes pratiques d'accréditation des commissions canadiennes de relations de travail et les problèmes auxquels elles font face dans l'accomplissement de leur devoir.

Quant au contenu du présent travail, disons immédiatement que cette étude comporte deux grandes parties: les commissions doivent déterminer la composition, le type et l'étendue des unités qu'elles accréditent et pour ce faire, elles ont recours à une série de critères qui sont comparés et évolués dans la première partie de cet ouvrage.

La partie principale de ce livre, la deuxième, est consacrée à l'étude des pratiques d'accréditation des commissions provinciales et fédérale. On y traite des problèmes tels lc composition, le type et l'étendue des unités de négociation, les différentes politiques concernant le métier, la sécurité, la poie horaire, les employés saisonniers et temporaires et finalement les attitudes vis-à-vis les unités couvrant plusieurs établissements et plusieurs employeurs. En plus, on y con- 